We add that the function

$$
\sigma^{*}(x, y)=\operatorname{Sup}_{m, n}\left|\sigma_{m, n}(x, y)\right|
$$

satisfies theorems analogous to Theorems 1,2 and 5 . The same may be said of the Abel and $(C, \alpha, \beta)(\alpha>0, \beta>0)$ means.

Corrigenda to the paper "On the differentiability of multiple integrals" by A. Z y g mund (Fundamenta Mathematicae, vol. 23, p. 143-149).

Prof. Banach kindly called my attention to the fact that the proof of the lemma on p. 145 is incomplete, for the argument on p. 146 , line 15 , is valid in the case $k=l$ only. The proof may be completed in various ways, and, in particular, as follows.

We have to show, that, given any fanctions $h=h(x, y), k=k(x, y)$, the expression.

$$
\mu(u, v)=\iint_{S} L_{h}(x-u) L_{k}(y-v) d x d y
$$

satisfies an inequality $I_{q}[\mu] \leqslant A_{q}$, whore $A_{q}$ depends on $q$ only. In the first place, we observe that, given any function $g=g(x)$, the function $\lambda(u)=\int_{0}^{1} L_{g}(u-x) d x$ belongs to every $L^{q}$, and the integral of $\lambda q(u)$ over $0 \leqslant u \leqslant 1$ does not exceed a constant $B_{q}^{q}$. This is an analogue, for the one-dimensional space, of the result which we have to prove; the proof follows by an argument similar to that of section 4 of the paper. Assuming this, let us consider any of the terms of the $\operatorname{sum} \underset{k, l=1}{\sum_{l=1}^{q}}\left\{I_{l=1}^{q}(k), \ldots \prod_{j=1}^{q}(l) \ldots\right\}$ on p. 146, line 9. Suppose first that $k$. $l=2$. Integrating first with respect to $x_{3}, \ldots, x_{q}, y_{3}, \ldots, y_{q}$, and then with respect to $x_{1}, x_{2}, y_{1}, y_{2}$, we obtain

$$
\int_{0}^{1} \int_{0}^{1} d x_{1} d y_{2} \mu^{q-2}\left(x_{1}, y_{2}\right) \int_{0}^{1} L_{2 h_{2}}\left(x_{1}-x_{2}\right) d x_{2} \int_{0}^{1} L_{2 k_{1}}\left(y_{1}-y_{1}\right) d y_{1} .
$$

Applying Hölder's inequality with the three exponents $q /(q-2), q$, $q$, we see that the integral does not exceed $I_{q}^{q-2}[\mu] B_{q}^{2}$. If $k=l$, e.g. $k=l=1$, the integra] is equal to $\int_{0}^{1} \int_{0}^{1} \mu^{q-1}\left(x_{1}, y_{1}\right) d x_{1} d y_{1} \leqslant I_{q}^{q-1}[\mu]$. Collecting the terms, we finally obtain

$$
I_{q}^{q}[\mu] \leqslant C_{q}\left\{I_{q}^{q-1}[\mu]+I_{q}^{q-2}[\mu]\right\},
$$

where $C_{q}$ depends on $q$ only. It is plainly sufficient to consider the case when $h(x, y)$ and $k(x, y)$ have a positive minimum. Then $I_{q}[\mu]$ is finite, and so does not exceed the largest root of the equation $t q-C_{q}\left(t q-1+t^{q-2}\right)=0$. This complotes the proof.

\section{On the strong derivatives of functions of intervals.}

By

\section{S. Saks (Warszawa).}

Introduction. Given a set of $2 p$ numbers $a_{1} \leqslant b_{1}, a_{2} \leqslant b_{2}, \ldots$, $a_{p} \leqslant b_{p}$ the set of points $\left(x_{1}, x_{2}, \ldots, x_{p}\right)$ such that $a_{i} \leqslant x_{i} \leqslant b_{i}$ for $i=1,2, \ldots, p$, will be denoted as the in terval $\left[a_{1}, b_{1} ; a_{2}, b_{2} ; \ldots ; a_{p}, b_{p}\right]$ of the $p$-dimensional space $\Re_{p}$. If $F(I)$ is an addditive function of intervals and $I_{0}$ an interval in $\Re_{p}$, then $\mathrm{V}\left(F ; I_{0}\right)$ will denote the total (absolute) variation of $F$ over $I_{0}$. If $F(I)$ is a function of intervals of bounded variation then it may be extended as a completely addditive function of sets to the family of all sets measurable $\left.{ }^{1}\right)(B)$; accordingly, in this case, $\nabla(F ; A)$ for any set $A$ measurable $(B)$ will mean the total variation of $F$ over $A$.

If $\left(x_{1}, x_{2}, \ldots, x_{p}\right)$ is a point in the space $\Re_{p}$ and $F(I)$ a function of intervals, then the lower and upper limits of the quotient $F(I) /$ meas $I$, where $I$ is an arbitrary interval containing $\left(x_{1}, x_{2}, \ldots, x_{p}\right)$ and $\mathrm{d}(I) \rightarrow 0$, will be called the lower and upper strong derivates of $F(I)$ at the point $\left(x_{1}, x_{2}, \ldots, x_{p}\right)$, and denoted by $F^{*}\left(x_{1}, x_{2}, \ldots, x_{p}\right)$ and $\bar{F}^{*}\left(x_{1}, x_{2}, \ldots, x_{p}\right)$ respectively. In the case when they are equal we shall write $F^{*}\left(x_{1}, x_{2}, \ldots, x_{p}\right)$ for their common value, that will be called the strong derivativea) of $F(I)$ at the point considered. $F^{\prime}\left(x_{1}, x_{2}, \ldots, x_{p}\right)$ will as usually denote the derivative of $F(I)$ in the ordinary sense. In the case

1) See for instance de la Vallée-P oussin, Intégrales de Lebesgue, Fonctions d'ensemble, Classes de Baire, 2。 éd., Paris (1934), pp. 88-95; Saks, Théorie de l'intégrale, Warszawa (1933), p. 250.

3) Some problems concerning the strong derivation of additive functions of intervals have been recently diseussed in a series of papers published in these Fundamenta; see the list at the ond of this note. 
$p=1$ both methods of derivation, ordinary and strong, are completely identical. In the case $p \geqslant 2$, however, they essentially differ and the existence of the ordinary derivative does not in general imply that of the strong one. In a former paper ${ }^{8}$ ) we have set an example of an additive non-negative and absolutely continuous function of intervals $\Phi(I)$ whose upper strong derivate is everywhere equal to $\infty$. In the first part of this note we attempt to complete this result as follows:

A. Given an arbitrary function $\sigma(t)>0$ in $[0, \infty]$ such that $\liminf _{t \rightarrow \infty} \sigma(t)=0$ there exists in the interval $S_{0}^{\prime}=[0,1 ; 0,1 ; \ldots ; 0,1]$

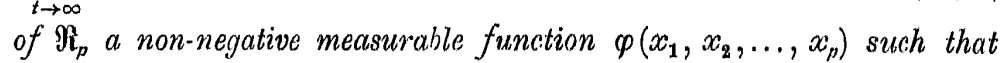
$\sigma(|\varphi|)|\varphi| \log ^{+\infty-1}|\varphi|$ is summable in $S_{0}$ and that for the indefinite integral $\Phi(I)$ of $\varphi$ the equation $\Phi^{*}\left(x_{1}, x_{2}, \ldots, x_{p}\right)=+\infty$ holds everywhere in $S_{0}$.

In a sense, this is the best possible result. For by a remarkable theorem of Jessen, Marcinkiewicz and Zygmund 4 , if $|\varphi| \log ^{+} p-1|\varphi|$ is integrable then the indefinite integral $\Phi(I)$ of $\varphi$ is strongly derivable almost everywhere and therefore $\Phi^{*}=\Phi^{\prime}$ almost everywhere in $\mathfrak{R}_{p}$. However, from another point of view, it remains yet to decide whether the condition $\bar{\Phi}^{*}=\infty$ in Theorem A might be replaced by a more complete one, viz. by $\mathscr{W}^{*}<\bar{\Phi}^{*}=\infty$. This may be done rather easily if we merely require the integrability of $\varphi$. A more serious difficulty seems to consist in the demand that $\sigma(|\varphi|)|\varphi| \log ^{+} p-1|p|$ should be integrable.

In the second part which is independent of the first the following generalization of a theorem of $\mathrm{Lu}$ s in will be established.

B. If $\varphi\left(x_{1}, x_{2}, \ldots, x_{p}\right)$ is a measurable function in the interval $S_{0}=[0,1 ; 0,1 ; \ldots ; 0,1]$, then for every $\varepsilon>0$ there exists an additive continuous function of intervals $\Phi(I)$ such that

(a) $\Phi^{*}\left(x_{1}, x_{2}, \ldots, x_{n}\right)=\varphi\left(x_{1}, x_{2}, \ldots, x_{n}\right)$ almost everywhere in $S_{0}$,

$$
V\left(\Phi ; S_{0}\right) \leqslant(1+\varepsilon) \int \ldots \int_{S_{0}}|\varphi| d x_{1} \ldots d x_{p} .
$$

Condition (b) is immaterial if $\varphi$ is not summable in $S_{0}$. In the case, however, when $\varphi$ is a summable function, it means that $\Phi(I)$ is

\footnotetext{
3) Saka, 6; seo also Busemann wollor, 2
}

4) Jessen, Marcinkiewicz and $z y g m u n d, 3$. of a bounded variation. More precisely, Theorem B may in that case be stated as follows:

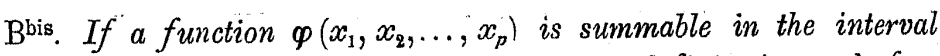
$S_{0}=[0,1 ; 0,1 ; \ldots ; 0,1]$ and $F(I)$ denotes the indefinite integral of $\varphi$, then, for every $\eta>0$, there exists an additive continuous and singular ${ }^{5}$ ) function of intervals $\Delta(I)$, such that $\mathrm{V}\left(\Delta ; S_{0}\right) \leqslant \eta$ and that the function $\Phi(I)=F(I)+\Delta(I)$ satisfies the condition (a) of Theorem $\mathrm{A}$.

Indeed, let $V_{0}$ denote the integral of $|\varphi|$ over $S_{0}$ and let $\varepsilon=\eta / V_{0}$ (we may obviously admit $V_{0}>0$ ). Let $\Phi(I)$ be a continuous additive function of intervals subject to the conditions (a) and (b) of Thecrem $A$, and let $\Delta(I)=\Phi(I)-F(I) . A s \Delta^{\prime}=\Phi^{\prime}-F^{\prime \prime}=\Phi^{*}-\varphi=0$ almost everywhere in $S_{0}$, the function $\Delta$ is a singular one. Thus there exists a set of measure zero $E$ in $S_{0}$ such that $\left.{ }^{6}\right) \mathrm{V}\left(\Delta ; S_{0}\right)=$ $=\mathrm{V}(\Delta ; E)$. Consequently

$$
\mathrm{V}\left(\Phi ; S_{0}\right)=\mathrm{V}(\Phi ; E)+\mathrm{V}\left(\Phi ; S_{0}-E\right)=\mathrm{V}(\Delta ; E)+\mathrm{V}\left(F ; S_{0}\right)=
$$$$
=\mathrm{V}\left(\Delta ; S_{0}\right)+V_{0}
$$

and so, in virtue of $(\mathrm{b})$, we get $\mathrm{V}\left(\Delta ; S_{0}\right) \leqslant \varepsilon V_{0}=\eta$, which proves the result.

For the cuse of one variable ( $p=1$ ), Theorem A was established by Lusin ?). In that case the condition (b) is naturally omitted as a superfluous one, since in $\mathfrak{l}_{1}$ the condition (a) is satisfied by the indefinite integral $\Phi(x)$ of the given function $\varphi(x)$ whenever the latter is summable in $[0,1]$. The same remark applies to the case of a space $\mathfrak{l}_{p}$ for arbitrary $p$ provided that the ordinary derivative is considered instead of the strong one.

For the sake of simplicity we shall consider the case of the plane. The reasonings are actually the same in the general case. Thus, in the sequel, by an interval $[a, b ; c, d]$ we shall mean the rectangle whose sides are parallel to the coordinate axes and whose opposite corners, lower left hand and upper right-hand, are

5) An additive function of intervals is said to be singular if it is of bounded variation and has the derivative (in the ordinary sense) almost everywhere equal to zero.

в) Cf. e. g. de la Vallée-Poussin, 1. c. ${ }^{1}$ ), pp. 102-112; Saks, 1. c. 1), pp. $256-257$.

7) Luвin, Integral and Trigonometrical Series (in Russian), Moscow (1915), pp. 34-41; Sur la notion de l'intégrale, Annali di Mrat. (3), t. 26 (1917), pp. 77-129. 
$(a, c)$ and $(b, d)$ respectively. Given a function $F(x, y)$ of two variables, the corresponding function of intervals will be denoted by the same letter; i. e. we shall put $F(l)=F(b, d)-F(a, d)-F(c, d)+$ $+F(a, b)$ for $I=[a, b ; c, d]$.

\section{I.}

1. Bohr construction. We shall recall some details of a $\mathrm{B} \circ \mathrm{hr} \mathrm{r}^{8}$ construction upon which the proof of Theorem A actually rests.

Let $S=[a, b ; c, d]$ be an interval, $\alpha>1$ an arbitrary number and $N=\mathrm{E} \alpha$. Consider a finite set of subintervals in $S$

(1.1) $I_{j}^{(1)}=\left[a, a+j \cdot \frac{b-a}{N} ; \quad c, c+\frac{d-c}{j}\right]$ where $j=1,2, \ldots, N$

Let us put

We have

$$
\Delta^{(1)}=\sum_{i=1}^{N} I_{j}^{(1)}, \quad \delta^{(1)}=\prod_{i=1}^{N} I_{i}^{(1)}
$$

meas $L_{j}^{(1)}=N$ meas $\delta_{j}^{(1)} \quad$ for $j=1,2, \ldots, N$,

meas $\Delta^{(1)}=N\left(1+\frac{1}{2}+\ldots+\frac{1}{N}\right)$ meas $\delta^{(1)} \geqslant \frac{N \log N}{2}$ meas $\delta^{(1)}$.

The remaining part of $S$, viz. $S-\Delta^{(1)}$, will be divided into a finite system of non-overlapping intervals. To each of them we apply the same operation as above to $S$. We carry out this process a sufficient number of times till the area of the remainder is less than (meas $S) /(N+1)^{2}$. Then we divide the remainder into a finite number of arbitrary non-overlapping intervals, $J^{(1)}, J^{(2)}, \ldots, J^{(r)}$, say. So we obtain a subdivision of $S$ into a set of non-overlapping intervals

$$
I_{1}^{(1)}, \ldots, I_{N}^{(1)} ; \quad I_{1}^{(2)}, \ldots, I_{N}^{(2)} ; \ldots ; \quad I_{1}^{(s)}, \ldots, I_{N}^{(s)} ; \quad J^{(1)}, J^{(2)}, \ldots, J^{(r)}
$$

such that, upon putting

we have

$$
\Delta^{(i)}=\sum_{j=1}^{N} I_{j}^{(i)}, \quad \delta^{(i)}=\prod_{j=1}^{N} I_{j}^{(i)} \quad \text { for } \quad i=1,2, \ldots, s,
$$

(1.2) meas $l_{j}^{(i)}=N$ meas $\delta^{(i)}$ for $i=1,2, \ldots, s ; j=1,2, \ldots, N$;

8) See Carathéodory, Vorlesungen ilber reelle Ifunktionen, 2 Aufl., Leipzig (1927), p. $689-691$

$$
\begin{gathered}
\text { meas } \Delta^{(i)} \geqslant \frac{1}{2} N \log N \text { meas } \delta^{(i)} \text { for } i=1, \ldots, s ; \\
\text { meas }\left(J^{(1)}+J^{(2)}+\ldots+J^{(r)}\right) \leqslant \frac{\text { meas } S}{(N+1)^{2}} .
\end{gathered}
$$

In the above subdivision of $S$ we have started from a set $(1,1)$ of intervals with a common corner at $(a, c)$ and their opposite corners on the curre $(x-a)(y-c)=$ $=(b-a)(d-c) / N=($ meas $S) / N$. So the total area $\Delta(1)$ of these intervals was approximately equal to

$$
\frac{\operatorname{meas} S}{N} \int_{(b-a) / N}^{b-a} \frac{d x}{x}=\frac{\log N}{N} \text { meas } S .
$$

If, however, instead of an interval on the plane we considered an interval $S=\left[a_{1}, b_{1} ; a_{2}, b_{2} ; \ldots, a_{p}, b_{p}\right]$ in a space $\mathfrak{R}_{p}$, then we should have to start $\mathrm{B} 0 \mathrm{hr}$ r's construction from a set of $p$-dimensional intervals with the common corner $\left(a_{1}, a_{2}, \ldots, a_{p}\right)$ and the opposite corners belonging to a $(p-1)$-dimensional variety $\left(x_{1}-a_{1}\right)\left(x_{2}-a_{2}\right) \ldots\left(x_{p}-a_{p}\right)=($ meas $S) / N^{p-1}$. The total volume of these intervals, as we easily compute, is approximately equal to $\left(\frac{\log N}{N}\right)^{p-1} \cdot$ meas $S$, while their product is an interval of volume (meas $S$ ) / $N^{p-1}$. Accordingly, passing to a apace $\mathfrak{R}_{p}$ we merely have to replace $N \log N$ in (1.3) by $N \log ^{p-1} N$ (with a suitable constant coefficient) and $(N+1)^{2}$ in $(1.4)$ by $(N+1)^{p}$. This explains the rôle of the $(p-1)$-st power of $\log |\varphi|$ in the general enunciation of Theorem A.

Now let $\psi(x, y)$ be the function equal to $\alpha$ over the set $\sum_{l=1}^{s} \delta^{(i)}+\sum_{i=1}^{r} J^{(i)}$, and to 0 elsewhere. In virtue of (1.3)

$$
\sum_{i=1}^{s} \text { meas } \delta^{(i)} \leqslant \frac{8}{(N+1) \log (N+1)} \sum_{i=1}^{s} \text { meas } \Delta^{(i)} \leqslant \frac{8 \text { meas } S}{\alpha \log \alpha} .
$$

Hence, by (1.4)

$\iint_{P} \psi \log \psi d x d y=\alpha \log \alpha \cdot\left(\sum_{i=1}^{s}\right.$ meas $\delta^{(i)}+\sum_{i=1}^{r}$ meas $\left.J^{(i)}\right) \leqslant 9$ meas $S$. On the other hand, it results from (1.2) and the definition of $\psi(x, y)$ that

$\iint_{l_{j}^{(i)}} \psi d x d y=\alpha \operatorname{meas} \delta^{(i)} \geqslant \operatorname{meas} I_{j}^{(i)}$ where $i=1,2, \ldots, s ; j=1,2, \ldots, N$

$$
\iint_{J^{(n)}} \psi d x d y=\alpha \operatorname{meas} J^{(i)} \quad \text { where } i=1,2, \ldots, r
$$


From the above discussion there follows

Lemma. Given an interval $S$ and a number $\alpha>1$ there exists in $S$ a function $\psi_{S, \alpha}(x, y)$ which enjoys the following properties:

$\left(\mathrm{A}_{1}\right) \psi_{s, \alpha}$ takes on two values only 0 and $\alpha$, each on a finite aggregate of intervals ${ }^{9}$ );

$\left(\mathrm{A}_{2}\right) \iint_{P} \psi_{S, \alpha}(x, y) \stackrel{+}{\log } \psi_{S, \alpha}(x, y) d x d y \leqslant 9$ meas $S$

$\left(\mathrm{A}_{8}\right)$ every point $(x, y)$ in $S$ belongs to an interval $I C S$ such that $\iint_{0} \psi_{s, \alpha}(x, y) d x d y \geqslant \operatorname{meas} l$.

2. Proof of Theorem A. Let now $\sigma(t)>0$ be a function in $[0,1]$ such that liminf $\sigma(t)=0$, and let $\left\{t_{t}\right\}$ bo an increasing sequence of values of $t$ such that $t_{t} \rightarrow \infty, \sigma\left(t_{i}\right) \rightarrow 0$. Denote by $\varepsilon(t)$ a continuous, non-decreasing function in $[0,1]$, which coincides with $\sigma(t)$ at the points $t_{i}$. We can obviously assume that $\varepsilon(t)<1$.

We shall define a sequence of non-negative functions $\left\{\psi_{k}(x, y)\right\}$ in the square $S_{0}=[0,1 ; 0,1]$ so as to satisfy the following conditions:

$\left(\mathrm{C}_{1}\right) \psi_{k}$ takes on a finite number of values only, say $0=\alpha_{0}^{(k)}<$ $<\alpha_{1}^{(k)}<\ldots<\alpha_{n_{k}}^{(k)}$ each of which over an aggregate of a finite number of intervals; $\psi_{0} \equiv 3$ identically;

$\left(\mathrm{C}_{2}\right) \quad \varepsilon\left(\alpha_{1}^{(k)}\right)<1 / 2^{k}, \quad \alpha_{1}^{(k)}>\sum_{j=1}^{k-1} \sum_{i=1}^{n_{j}-\mu^{-1}} \alpha_{i}^{(j)}$ for $k_{i} \geqslant 1$ :

$\left(\mathrm{C}_{3}\right)$ the sum

$$
\frac{\psi_{0}(x, y)}{\left[\varepsilon\left(\alpha_{1}^{(0)}\right)\right]^{1 / 2}}+\frac{\psi_{1}(x, y)}{\left[\varepsilon\left(\alpha_{1}^{(2)}\right)\right]^{1 / 2}}+\ldots+\frac{\psi_{k}(x, y)}{\left[\varepsilon\left(\alpha_{1}^{(k)}\right)\right]^{1 / 2}}
$$

admits only the values which belong to the set $\left\{t_{i}\right\}$;

$\left(\mathrm{C}_{\text {k }}\right) \quad \iint_{S_{0}} \psi_{k} d x d y \leqslant \iint_{S_{0}} \psi_{k} \log ^{+} \psi_{k} d x d y \leqslant 9 ;$

$\left(\mathrm{C}_{5}\right)$ every point $(x, y)$ in $S$ belongs to an interval $I \subset S_{0}$ such that $\mathrm{d}(I)<1 / k$ and $\iint_{i} \psi_{k} d x d y \geqslant$ meas $l$.

D) We neglect sets of points of measure zero.
For suppose that $\psi_{k}$ are defined and satisfy the above conditions for $k \leqslant h-1$. Divide $S_{0}$ into a finite number of intervals $I_{1}, I_{2}, \ldots, I_{n_{n}}$ so that the sum $\sum_{j=0}^{h-1} \psi_{j}(x, y) /\left[\varepsilon\left(\alpha_{1}^{(j)}\right)\right]^{1 / 2}$ has a constant value over each of them, say $\beta_{n}$ over $I_{n}$ for $n=1,2, \ldots, n_{n}$. This may obviously be done in virtue of the condition $\left(\mathrm{C}_{1}\right)$ which by assumption is satisfied for $k=1,2, \ldots, h-1$. We can admit that $I_{n}$ are of diameters less than $1 / h$.

Now a number $\alpha_{1}^{(h)}$ will be chosen sufficiently large so as to satisfy the condition $\left(\mathrm{C}_{2}\right)$ for $k=h$. Since $t /[\varepsilon(t)]^{1 / 2}$ is continuous and tends with $t$ to $+\infty$, the value of $\alpha_{1}^{(h)}$ may be determined in such manner that $\beta_{1}+\alpha_{1}^{(h)} /\left[\varepsilon\left(\alpha_{1}^{(h)}\right)\right]^{1 / 2}$ belong to the set $\left\{t_{i}\right\}$. Next the numbers $\alpha_{n}^{(h)}$ for $n=2, \ldots, n_{h}$ will be defined so that $\alpha_{1}^{(h)}<$ $<\alpha_{2}^{(h)}<\ldots<\alpha_{n_{h}}^{(h)}$ and that the values $\beta_{n}+\alpha_{n}^{(h)} /\left[\varepsilon\left(\alpha_{1}^{(h)}\right)\right]^{1 / 2}$ all belong to the set $\left\{t_{t}\right\}$. Thus upon putting $\psi_{h}(x, y)=\psi_{I_{n}}, \alpha_{n}^{(h)}(x, y)$ on $I_{n}$ for $n=1,2, \ldots, n_{h}$, where $\psi_{I_{n}, \alpha_{n}^{(h)}}$ is a function satisfying the conditions of the lemma of $\S 1$ with $S=I_{n}$ and $\alpha=\alpha_{n}^{(h)}$, it is readily seen that $\psi_{h}(x, y)$ and $\alpha_{1}^{(h)}, \alpha_{2}^{(h)}, \ldots, \alpha_{n_{h}}^{(h)}$ verify all the conditions $\left(\mathrm{C}_{1}\right),\left(\mathrm{C}_{2}\right)$, $\left(\mathrm{C}_{3}\right),\left(\mathrm{C}_{4}\right)$ and $\left(\mathrm{C}_{5}\right)$ for $k=h$.

We now turn to the proof of Theorem A. Let us set

$$
\varphi(x, y)=\sum_{k=0}^{\infty} \frac{\psi_{k}(x, y)}{\left[\varepsilon\left(\alpha_{1}^{(k)}\right)\right]^{1 / 2}}
$$

and let $\varphi_{n}(x, y)$ denote the $n$th partial sum of $\varphi(x, y)$. Let

$$
R_{n}=\underset{(x, y)}{\mathrm{E}}\left[\varphi_{n}(x, y)>\varphi_{n-1}(x, y)\right]=\underset{(x, y)}{\mathrm{E}}\left[\psi_{n}(x, y) \geqslant \alpha_{1}^{(n)}\right] .
$$

It results from $\left(\mathrm{C}_{1}\right)$ and $\left(\mathrm{C}_{2}\right)$ that over $R_{n}$ we have

$$
\varphi_{n-1}(x, y) \leqslant \sum_{k=0}^{n-1} \frac{\alpha_{n_{k}}^{(k)}}{\left[\varepsilon\left(\alpha_{1}^{(n)}\right)\right]^{1 / 2}}<\frac{\alpha_{1}^{(n)}}{\left[\varepsilon\left(\alpha_{1}^{(n)}\right)\right]^{1 / 2}} \leqslant \frac{\psi_{n}(x, y)}{\left[\varepsilon\left(\alpha_{1}^{(n)}\right)\right]^{1 / 2}} ;
$$

whence $\varphi_{n}(x, y) \leqslant 2 \psi_{n}(x, y) /\left[\varepsilon\left(\alpha_{1}^{(n)}\right)\right]^{1 / 2}$ for $(x, y) \varepsilon R_{n}$, and in virtue of $\left(\mathrm{C}_{4}\right)$

(2.1) $\iint_{R_{n}} \varphi_{n} \log \varphi_{n} d x d y \leqslant \frac{2}{\left[\varepsilon\left(\alpha_{1}^{(n)}\right)\right]^{1 / 2}}\left\{\iint_{R_{n}} \psi_{n} \log \psi_{n} d x d y+\right.$
$\left.+\log \frac{2}{\left[\varepsilon\left(\alpha_{1}^{(n)}\right)\right]^{1 / 2}} \iint_{R_{n}} \psi_{n} d x d y\right\} \leqslant \frac{18}{\left[\varepsilon\left(\alpha_{1}^{(n)}\right)\right]^{1 / 2}}\left(1+\log \frac{2}{\left[\varepsilon\left(\alpha_{1}^{(n)}\right)\right]^{1 / 2}}\right)$.
Fandamenta Mathematicae T. Xxv. 
Thus, since by $\left(\mathrm{C}_{3}\right)$ the valucs of $\varphi_{n}(x, y)$ belong to the set $\left\{t_{l}\right\}$ we have $\sigma\left[\varphi_{n}(x, y)\right]=\varepsilon\left[\varphi_{n}(x, y)\right] \leqslant \varepsilon\left(\alpha_{1}^{(n)}\right)$ for $(x, y) \in R_{n}$, and therefore, it follows from (2.1) that

$$
\iint_{R_{n}} \varepsilon\left(\varphi_{n}\right) \varphi_{n} \log \varphi_{n} d x d y \leqslant \varepsilon_{n},
$$

where $\varepsilon_{n}$ has been written for $18\left[\varepsilon\left(\alpha_{1}^{(n)}\right)\right]^{1 / 2}\left(1+\log 2 /\left[\varepsilon\left(\alpha_{1}^{(n)}\right)\right]^{1 / 2}\right) \cdot \operatorname{In}$ virtue of the first of the relations $\left(\mathrm{C}_{2}\right)$ we have

$$
\sum_{n=1}^{\infty} \varepsilon_{n}<+\infty
$$

Now let $E_{n}=\underset{(x, y)}{\mathbf{E}}\left[\varphi(x, y)=\varphi_{n}(x, y)>\varphi_{n \sim 1}(x, y)\right]$. As it results from $\left(\mathrm{C}_{4}\right)$ and $\left(\mathrm{C}_{1}\right)$ we have $\psi_{k}(x, y)=0$ everywhere in $S_{0}^{\gamma}$ with the exception at most over a set of measure less than $9 / \alpha_{1}^{(k)}$. Hence, since by the second of the relations $\left(\mathrm{C}_{2}\right)$ the series $\sum_{k} \frac{1}{\alpha_{1}^{(k)}}$ apparently converges, we have $S_{0}=E+\underset{n}{\Sigma} E_{n}$ where $E$ is a set of measure zero. As $E_{n} \subset R_{n}$, from (2.2) and (2.3) we infer thus

$$
\text { (2.4) } \begin{gathered}
\iint_{S_{0}} \varepsilon(\varphi) \varphi \log \varphi d x d y \leqslant \sum_{n=1}^{\infty} \iint_{E_{n}} \varepsilon\left(\varphi_{n}\right) \varphi_{n} \log \varphi_{n} d x d y \leqslant \\
\leqslant \sum_{n=1}^{\infty} \varepsilon_{n}<\infty .
\end{gathered}
$$

On the other hand, it follows from $\left(\mathrm{C}_{5}\right)$ that for any $k_{0}$ there corresponds to every point $(x, y)$ in $S_{0}$ an interval $I \subset S_{0}$ such that $(x, y) \in I, \mathrm{~d}(I)<1 / k$ and

$$
\iint_{I} \varphi d x d y \geqslant \frac{1}{\varepsilon\left(\alpha_{1}^{(k)}\right)} \iint_{I} \psi_{k} d x d y \geqslant \frac{\operatorname{meas} I}{\varepsilon\left(\alpha_{1}^{(k)}\right)} .
$$

Since $\varepsilon\left(\alpha_{1}^{(k)}\right) \rightarrow 0$ as $k \rightarrow \infty$, this means that the upper strong derivate of the indefinite integral of $\varphi(x, y)$ is $\infty$ everywhere in $S_{0}$. Hence in view of (2.4) the function $\varphi(x, y)$ satisfies both conditions of Theorem A.

\section{II.}

1. Lemmas. The proof of Theorem B will rest on a few elementary lemmas.

Lemma 1. Given a linear function $F^{\prime}(x)=p x+q$ in an interval $[a, b]$ and positive numbers $\varepsilon, \eta<1$, there exist a monotonic continuous function $\Phi(x)$ in $[a, b]$ and $a$ set $P$ of measure $\eta \cdot(b-a)$ such that

(1.3) $\left|\Phi\left(x^{\prime}\right)-\Phi(x)\right| \leqslant \frac{4|p|}{\eta}\left|x^{\prime}-x\right|$ for $x^{\prime} \in[a, b], \quad x \in[a, b]-P$.

Proof. Divide $[a, b]$ into $n$ intervals $I_{1}, I_{2}, \ldots, I_{n}$ of equal length $\delta=(b-a) / n<\varepsilon /|p|$. Next, divide each interval $I_{k}=$ $=[a+(k-1) \delta, a+k \delta]$ into five sub-intervals, viz.

$$
\begin{gathered}
J_{k}^{(1)}=\left[a+(k-1) \delta, a+\left(k-1+\frac{\eta}{4}\right) \delta\right], \\
J_{k}^{(2)}=\left[a+\left(k-1+\frac{\eta}{4}\right) \delta, a+\left(k-1+\frac{\eta}{2}\right) \delta\right], \\
J_{k}=\left[a+\left(k-1+\frac{\eta}{2}\right) \delta, a+\left(k-\frac{\eta}{2}\right) \delta\right], \\
J_{k}^{(3)}=\left[a+\left(k-\frac{\eta}{2}\right) \delta, a+\left(k-\frac{\eta}{4}\right) \delta\right], \\
J_{k}^{(4)}=\left[a+\left(k-\frac{\eta}{4}\right) \delta, a+k \delta\right] .
\end{gathered}
$$

The intervals $J_{k}^{()}$(where $k=1,2, \ldots, n ; j=1,2,3,4$ ) are of equal length $\eta \delta / 4$, and so, upon denoting their sum by $P$, we have meas $P=4 n \eta \delta / 4=\eta \cdot(b-a)$.

Now let $\Phi(x)$ denotes a continuous and monotonic function in $[a, b]$ which has the derivative $\Phi^{\prime}(x)$ almost everywhere equal to 0 , coincides with $F(x)$ at the middle- and end-points of the intervals $I_{k}$ and is constant in the intervals $J_{k}^{(2)}+J_{k}+J_{k}^{(3)}$. Since the oscillation of $\Phi(x)-F(x)$ over each interval $I_{k}$ is less than, or equal to, $|p| \boldsymbol{\delta}<\boldsymbol{\varepsilon}$ we see at once that the condition (1.1) is satisfied. In order to 
prove (1.3) we may evidently suppose that both points $x^{\prime}$ and $x$ belong to the same interval $I_{k}$, i. $\theta$. that $x \in J_{k}$ and $x^{\prime} \in I_{k}$. Then if $x^{\prime} \in J_{k}^{(2)}+J_{k}+J_{k}^{(3)}$ we have $\Phi\left(x^{\prime}\right)=\Phi(x)$ and the inequality in (1.3) is obvious. If, however, $x^{\prime} \in J_{k}^{(1)}+J_{k}^{(4)}$ then $\left|x^{\prime}-x\right| \geqslant \eta \delta / 4$ and, since $\left|\Phi\left(x^{\prime}\right)-\Phi(x) \leqslant\right| p \mid \delta$, we again obtain the inequality in (1.3).

Lemma 2. Let $\psi(x)$ be a measurable function in $[a, b]$ such that $|\psi(x)| \leqslant M<\infty$. Then for any pair of positive numbers $\varepsilon, \eta<1$ there exist a continuous function $\Psi(x)$ in $[a, b]$ and $a$ set $P$ of measure $\eta \cdot(b-a)$ such that

$$
|\Psi(x)| \leqslant \varepsilon \quad \text { in } \quad[a, b]
$$

$\Psi^{\prime}(x)=\psi(x)$ almost everywhere in $[a, b]$,

$\left|\Psi\left(x^{\prime}\right)-\Phi(x)\right| \leqslant \frac{5 M}{\eta}\left|x^{\prime}-x\right|$ whenever $x^{\prime} \in[a, b]$ and $x \in[a, b]-P$,

$$
\mathrm{V}(\Psi ; a, b) \leqslant 2 \int_{a}^{b}|\psi(x)| d x .
$$

Proof. Let $G(x)$ be un indefinite integral of $\psi(x)$ in $[a, b]$. Divide $[a, b]$ into a finite number of intervals $I_{1}, I_{2}, \ldots, I_{n}$ so that the oscillation of $G(x)$ be less than $\varepsilon / 2$ on euch of then. Let $F^{\prime}(x)$ denote the function which is linear in each of these intervals and coincides with $G(x)$ at their end-points. The angular coefficient of $F(x)$ in every interval $I_{k}$ is less than, or equal to $M$. Hence, by applying the preceding lemma to the function $F(x)$ in each of the intervals $I_{k}$, we obtain a set $P$ of measure $\eta \cdot(b-a)$ and a function $\Phi(x)$, continuous in $[a, b]$ and monotonic in each $I_{k}$, such that

$$
|\Phi(x)-F(x)|<\varepsilon / 2 \text { in }[a, b], \quad \mathrm{V}\left(\Phi ; I_{k}\right)=\left|\int_{l_{k}} \psi(x) d x\right| \text {, }
$$

$$
\Phi^{\prime}(x)=0 \text { almost everywhere in }[a, b] \text {, }
$$

(1.6) $\left|\Phi\left(x^{\prime}\right)-\Phi(x)\right| \leqslant \frac{4 M}{\eta}\left|x^{\prime}-x\right|$ whenever $x^{\prime} \in[a, b]$ and $x \in[a, b]-P$.

Let $\Psi(x)=G(x)-\Phi(x)$. Since $|G(x)-F(x)| \leqslant \varepsilon / 2$ throughout the interval $[a, b]$, and $\left|G\left(x^{\prime \prime}\right)-G\left(x^{\prime}\right)\right| \leqslant M \cdot\left|x^{\prime \prime}-x^{\prime}\right|$ for any pair of points $x^{\prime}, x^{\prime \prime}$ in $[a, b]$, we conclude at once from (1.4), (1.5) and (1.6) that the function $\Psi(x)$ together with the set $P$ satisfy the required conditions of the lemma.
Lemma 3. Let $f(x, y)$ be a measurable function, $E$ a closed set in the square $S_{0}=[0,1 ; 0,1]$ and $\sigma<1$ a positive number. Then there exist in $S_{0}$ an additive continuous function of intervals $F(I)$ and a measurable set $Q$ such that

Proof. We can approximate $f(x, y)$ by a function

$$
f_{0}(x, y)=\sum_{k=1}^{n} g_{k}(x) h_{k}(y)
$$

where $h_{k}(x)$ and $g_{k}(x)$ are continuous functions in $[0,1]$, so that $\left.{ }^{10}\right)$

$$
\begin{gathered}
\iint_{s_{0}}\left|f(x, y)-f_{0}(x, y)\right| d x d y<\frac{\sigma^{2}}{2}, \\
\sum_{k=1}^{n} \iint_{s_{0}}\left|g_{k}(x) h_{k}(y)\right| d x d y \leqslant \iint_{s_{0}}|f(x, y)| d x d y .
\end{gathered}
$$

On the other hand, an aggregate of non-overlapping intervals $I_{1}, I_{2}, \ldots, I_{m}$ may be determined in $S_{0}-E$ so that

$$
\int_{S_{0}-E-R} \int_{E-R}|f(x, y)| d x d y<\frac{\sigma^{2}}{2} \quad \text { where } \quad R=\sum_{k=1}^{m} I_{k}
$$

10) In order to satiefy the condition (1.8) it is convenient to set the function $f(x, y)$ in the form $f=f_{1}-f_{2}$, where $f_{1} \geqslant 0, f_{2} \geqslant 0$ and $f_{r} \cdot f_{2}=0$, and then to apply the well-known methods of approximation to $f_{1}$ and $f_{3}$ separately. 
Let $M$ denote the upper bound of the values of $\left|g_{k}(x)\right|$ and $\left|h_{k}(x)\right|$ in $[0,1]$ and let $l=\mathrm{d}(E, R)>0$ be the distance of the sets $E^{\prime}$ and $R$. Then, in virtue of Lemma 2 a system of functions $G_{k}(x), H_{k}(x)$, where $k=1,2, \ldots, n$, and a set $P$ of linear measure less than $\sigma / 2$, may be defined in $[0,1]$ so as to satisfy the following conditions:

(1.10) $\quad\left|G_{k}(x)\right|<\frac{l \sigma^{2}}{160 M n^{2} m}, \quad\left|H_{k}(x)\right|<\frac{l \sigma^{2}}{160 M M n^{2} m} \quad$ in $[0,1]$

(1.11) $\quad G_{k}^{\prime}(x)=g_{k}(x), \quad H_{k}^{\prime}(x)=h_{k}(x) \quad$ almost everywhere in $[0,1]$

$$
\left\{\begin{array}{c}
\mid G_{k}\left(x^{\prime}\right)-\left(x_{k}^{x}(x) \mid \leqslant \frac{20 M n\left|x^{\prime}-x\right|}{\sigma}\right. \\
\left|H_{k}\left(x^{\prime}\right)-H_{k}(x)\right| \leqslant \frac{20 M n\left|x^{\prime}-x\right|}{\sigma} \\
\text { whenewer } x^{\prime} \in[0,1] \text { and } x_{i} \in[0,1]-P
\end{array}\right.
$$

(1.13) $\mathrm{V}\left(G_{k} ; 0,1\right) \leqslant 2 \int_{0}^{1}\left|g_{k}(x)\right| d x, \quad \mathrm{~V}\left(H_{k} ; 0,1\right) \leqslant 2 \int_{0}^{1}\left|h_{k}(x)\right| d x$.

Let 'us now put

$$
\text { (1.14) } \Psi(x, y)=\sum_{k=1}^{n} G_{k}(x) H_{k}(y), \quad H^{\prime}(I)=\sum_{k=1}^{m} \Psi\left(I \cdot I_{k}\right),
$$

and let $Q$ denote the plane set of points $(x, y)$ whose at least one coordinate belongs to $P$. It will be shown that the function $F(I)$ and the set $Q$ satisfy the conditions (i-vi).

Indeed, we have meas $Q \leqslant 2$ lin meas $P<\sigma$ i. e. the condition (i). Next, by (1.14), the function $F(I)$ vanishes for any interval outside of $R$, whence $F^{*}(x, y)=0$ identically over $S_{0}-R$, and in particular over the set $E$. Further, by (1.14) and (1.11)

$$
\Psi^{*}(x, y)=\sum_{k=1}^{n} G_{k}^{\prime}(x) H_{k}^{\prime}(y)=\sum_{k=1}^{n} g_{k}(x) h_{k}(y)=f_{0}(x, y) \text { almost everywhere }
$$

and therefore, on account of (1.14), (1.9) and (1.7), we have

$$
\int_{S_{0}-E} \int_{E}\left|F^{*}(x, y)-f(x, y)\right| d x d y \leqslant \int_{S_{0}-E} \int_{E} \mid H^{*}(x, y)-f_{0}(x, y) d x d y+
$$

$+\iint_{S_{0}}\left|f_{0}(x, y)-f(x, y)\right| d x d y \leqslant \iint_{R}\left|\Psi^{*}(x, y)-f_{0}(x, y)\right| d x d y+$
$\quad+\int_{S_{0}-E-R} \int_{E}\left|\Psi^{*}(x, y)\right| d x d y+\iint_{S_{0}}\left|f_{0}(x, y)-f(x, y)\right| d x d y<\sigma^{2}$

which means the condition (iii).

In order to prove the condition (iv) consider an arbitrary interval $I=[a, b ; c, d]$ such that $I \cdot(E-Q) \neq 0$. The inequality $F(I) \leqslant \sigma \cdot$ meas $I$ is obvious in case $I \cdot R=0$, for then $F(I)=0$. Hence suppose that $I \cdot R \neq 0$. Then $\mathrm{d}(I) \geqslant \mathrm{d}(E, R)=l$, and therefore one at least of the numbers $b-a, c-d$ is greater than $l / 2$. Suppose that $b-a \geqslant l / 2$. We may also suppose that one of the corners of $I$, say the point $(a, c)$, belongs to $E-Q$; for, otherwise, we might divide $I$ into four, or two, intervals so that their common corner should belong to $E-Q$.

Now let $J=[\alpha, \beta ; \gamma, \delta]$ be an arbitrary sub-interval of $I$. As, by assumption the point $(a, c)$ does not belong to $Q$, its ordinate $c$ certainly does not belong to the set $P$ and, in view of (1.12), we have $\left|H_{k}(\delta)-H_{k}(\gamma)\right| \leqslant\left|H_{k}(\delta)-H_{k}(c)\right|+\left|H_{k}(\gamma)-H_{k}(c)\right| \leqslant \frac{40 M n(d-c)}{\sigma}$ for $k=1,2, \ldots, n$. On the other hand, in virtue of (1.10)

$$
\left|G_{k}(\beta)-G_{k}(\alpha)\right| \leqslant \frac{l \sigma^{2}}{80 M n^{2} m} \leqslant \frac{\sigma^{2} \cdot(b-a)}{40 M n^{2} m} .
$$

Hence, for every interval $J=[\alpha, \beta ; \gamma, \delta] \subset I$

$$
\begin{gathered}
\Psi(J) \leqslant \sum_{k=1}^{n}\left|G_{k}(\beta)-G_{k}(\alpha)\right| \cdot\left|H_{k}(\delta)-H_{k}(\gamma)\right| \leqslant \\
\leqslant n \cdot \frac{\sigma^{2} \cdot(b-a)}{40 M n^{2} m} \cdot \frac{40 M n(d-c)}{\sigma} \leqslant \frac{\sigma \text { meas } I}{m} .
\end{gathered}
$$

Thus $|F(I)| \leqslant \sum_{k=1}^{m}\left|\Psi\left(I \cdot I_{k}\right)\right| \leqslant \sigma$ meas $I$, and so the condition (iv) is established. Further from (1.14), (1.13) and (1.8) we derive

$$
\begin{gathered}
\mathrm{V}\left(\boldsymbol{F} ; S_{0}\right)=\mathrm{V}(\Psi ; R) \leqslant \mathrm{V}\left(\Psi ; S_{0}\right) \leqslant \sum_{k=1}^{n} \mathrm{~V}\left(G_{k} ; 0,1\right) \cdot \mathrm{V}\left(H_{k} ; 0,1\right) \leqslant \\
\quad \leqslant 4 \sum_{k=1}^{n} \int_{0}^{1}\left|g_{k}(x)\right| d x \cdot \int_{0}^{1}\left|h_{k}(x)\right| d x \leqslant 4 \iint_{S_{0}}|f(x, y)| d x d y
\end{gathered}
$$


which proves the condition (v). Finally, (vi) apparently follows from (1.14) and (1.10).

2. Proof of Theorem B. Let $\varphi(x, y)$ be a measurable function in the square $S_{0}=[0,1 ; 0,1]$ and $\varepsilon<1$ a positive number. Let $V_{0}=\iint_{S_{0}}|\varphi(x, y)| d x d y$. For the sequel we may clearly suppose that $32 \varepsilon<V_{0}$. We shall define a non-decreasing sequence of closed sets $\left\{E_{n}\right\}$, a sequence of mesurable sets $\left\{Q_{n}\right\}$ and a sequence of continuous additive functions of intervals $\left\{F_{n}^{\prime}(L)\right\}$ so as to satisfy the following conditions

$$
\operatorname{meas}\left(S_{0} \cdots-E_{n}\right)<\frac{1}{2^{n-1}}
$$$$
\text { meas } Q_{n}<\frac{1}{2^{n}} \text {; }
$$

$\left(\mathrm{P}_{1}\right) \quad\left|F_{n}^{\prime}(I)\right|<\frac{1}{2^{n}}$ for $n>1$ and every interval $I C S_{0}$;

$\left(\mathrm{P}_{4}\right) F_{n}(I) \leqslant \frac{\text { meas } I}{2^{n}}$ whenever $n>1$ and $I \cdot\left(E_{n-1}-Q_{n}\right) \neq 0$;

$\left(\mathrm{P}_{5}\right) \quad \mathrm{V}\left(F_{n} ; S_{0}\right) \leqslant \frac{\varepsilon V_{0}}{2^{n}}$ for $n>1 ; \quad \mathrm{V}\left(H_{1}^{\prime} ; S_{0}\right) \leqslant V_{0} ;$

$\left(\mathrm{P}_{6}\right)\left|\sum_{n=1}^{n} F_{k}^{*}(x, y)-\varphi(x, y)\right|<\varepsilon^{2}$ on $E_{n}^{r} ; W_{n}^{*}(x, y)=0$ on $E_{n-1}^{\prime}$ for $n>1$

$\left(\mathrm{P}_{7}\right) \quad \int_{s_{0}-E_{n}}\left\{\int_{k=1}|\varphi(x, y)|+\sum_{k=1}^{n}\left|F_{k}^{*}(x, y)\right|\right\} d x d y \leqslant \frac{\varepsilon V_{0}}{2^{n+8}}$.

We proceed by induction. First, let $E_{0}$ be a closed set in $S_{0}$ such that $\varphi(x, y)$ is continuous over $E_{0}$ and that $\iint_{S_{0}-E_{0}}|\varphi| d x d y \leqslant \varepsilon V_{0} / 33$; let $F_{1}(I)=\iint \varphi(x, y) d x d y$. Since $\varphi$ is bounded over $E_{E_{0} \cdot X}$ we have $\dot{H}_{1}^{*}(x, y)=\varphi(x, y)$ at almost every point $\left.{ }^{11}\right)(x, y) \in E_{0}$. Let $E_{1}$ be a closed subset of $E_{0}$ such that $H_{1}^{*}(x, y)=\varphi(x, y)$ e ver y w here on $E_{1}$ and that $\iint_{S_{0}-E_{1}}|\varphi| d x d y \leqslant \varepsilon V_{0} / 32$. On putting $Q_{1}=0$, we see at once that $E_{1}, F_{1}(I)$ and $Q_{1}$, as defined above, satisfy the conditions
$\left(\mathrm{P}_{1}-\mathrm{P}_{7}\right)$ for $n=1$.

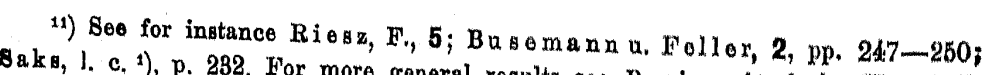
8aks, J. c. 1), p. 232. For more general results seo Besicovitch 1; Waxd, 7 .
Now let us suppose that $E_{n}, F_{n}(I)$ and $Q_{n}$ are defined and satisfy these conditions for $n \leqslant p-1$. Put

$$
f_{p}(x, y)=\varphi(x, y)-\sum_{k=1}^{p-1} F_{k}^{*}(x, y)
$$

In virtue of $\left(\mathrm{P}_{7}\right)$ for $n=p-1$ we have

$$
\iint_{S_{0}-E_{p-1}}\left|f_{p}(x, y)\right| d x d y \leqslant \frac{\varepsilon V_{0}}{2^{p+2}}
$$

Next, let $\sigma_{p}<\varepsilon^{2} / 2^{p}$ be a positive number such that, for any measurable set $A$ in $S_{0}$,

(2.2) $\iint_{A}\left\{|\varphi(x, y)|+\sum_{k=1}^{p-1}\left|F_{k}^{*}(x, y)\right|\right\} d x d y \leqslant \frac{\varepsilon V_{0}}{2^{p+5}}$ if meas $A \leqslant \sigma_{p^{*}}$ Let $Q_{p}=Q$ be a set and $F_{p}=F$ an additive continuous function, satisfying the conditions (i-vi) of Lemma 3 with $\sigma=\sigma_{p}$ $E=E_{p-1}$ and $f(x, y)=f_{p}(x, y)$. So we have

$$
\iint_{s_{0}-E_{p-1}}\left|F_{p}^{*}(x, y)-f_{p}(x, y)\right| d x d y<\sigma_{p}^{2}
$$

and therefore the inequality

$$
\left|\sum_{k=1}^{p} F_{k}^{*}(x, y)-\varphi(x, y)\right|=\left|F_{p}^{*}(x, y)-f_{p}(x, y)\right|<\sigma_{p}<\varepsilon^{2}
$$

holds everywhere on $S_{0}-E_{p-1}$, except at most on a subset of measure less than $\sigma_{p}$. Let $H_{p}$ denote a closed subset of $S_{0}-E_{p-1}$ such that (2.4) holds on it everywhere and that

$$
\text { meas }\left(S_{0}-E_{p-1}-H_{p}\right) \leqslant \sigma_{p} \text {. }
$$

Hence on putting $E_{p}=E_{p-1}+H_{p}$ we have

$$
\text { meas }\left(S_{0}-E_{p}\right) \leqslant \sigma_{p} \text {. }
$$

We shall show that $E_{p}, Q_{p}$ and $F_{p}$ satisfy the required conditions $\left(\mathrm{P}_{1}-\mathrm{P}_{7}\right)$ for $n=p$. Indeed, the condition $\left(\mathrm{P}_{1}\right)$ is already contained in (2.5). Next, for $\sigma=\sigma_{p}, Q=Q_{p}, f=f_{p}$ the conditions (i), 
(vi) and (iv) of Lemma 3 inolude the conditions $\left(P_{2}\right),\left(P_{8}\right)$ and $\left(P_{4}\right)$ respectively, while from (v), on account of (2.1), there follows

$$
V\left(f_{p}^{\prime} ; S_{0}\right) \leqslant 4 \int_{S_{0}-E_{p, 1}}\left|f_{p}(x, y)\right| d x d y \leqslant \frac{\varepsilon V_{0}}{2^{n}}
$$

which is the condition $\left(\mathrm{P}_{5}\right)$. Further, the equation $F_{n}^{*}(x, y)=0$ and the inequality (2.4) are everywhere satisfied over the sets $E_{p-1}^{\prime}$ and $H_{p}=E_{p}-E_{p-1}$ respectively; hence, as the relations $\left(\mathrm{P}_{\mathrm{B}}\right)$ hold by assumption for $n=p-1$, they are again true for $n=p$. Finally, by (2.5) and (2.2), we have

$$
\iint_{S_{0}-E_{p}}\left\{|\varphi(x, y)|+\sum_{k=1}^{p \cdots 1} \mid H_{k}^{*}(x, y)\right\} \mid d x d y \leqslant \frac{\varepsilon V_{0}}{2^{n+5}}
$$

whence, in virtue of $(2,3)$ and $\sigma_{p}^{2}<\varepsilon^{4} / 2^{2 p}<\varepsilon V_{0} / 2^{n} \cdot 32$,

(2.7) $\iint_{S_{0}-E_{p}}\left|F_{p}^{*}(x, y)\right| d x d y \leqslant \iint_{S_{0}-E_{p}}\left|f_{p}(x, y)\right| d x d y+\sigma_{p}^{2} \leqslant \frac{\varepsilon V_{0}}{2^{p+4}}$.

By adding the inequalities (2.6) and (2.7) we obtain the relation $\left(\mathrm{P}_{7}\right)$ for $n=p$.

Let us now put

$$
\begin{gathered}
F^{\prime}(I)=\sum_{k=1}^{\infty} F_{k}(I) \\
\psi(x, y)=\varphi(x, y)-\sum_{k=1}^{\infty} F^{*}(x, y), \quad \Psi(I)=\iint_{I} \psi d x d y \\
\Phi(I)=F^{\prime}(I)+\Psi(I) \\
A=\sum_{k=1}^{\infty} E_{k}-\prod_{n=1}^{\infty} \sum_{k=1}^{\infty} Q_{k} .
\end{gathered}
$$

In virtue of $\left(\mathrm{P}_{8}\right)$ the series (2.8) converges uniformly in $S_{0}$, and by $\left(\mathrm{P}_{5}\right)$ we have

(2.9) $\mathrm{V}\left(F ; S_{0}\right) \leqslant \mathrm{V}\left(H_{1} ; S_{0}\right)+\sum_{n=2}^{\infty} \mathrm{V}\left(F_{n} ; S_{0}\right) \leqslant\left(1+\frac{\varepsilon}{2}\right) \cdot V_{0}$.

On the other hand, it follows from $\left(\mathrm{P}_{\theta}\right)$ and $\left(\mathrm{P}_{1}\right)$ that almost everywhere in $S_{0}$ the series $\underset{k}{\sum} F_{k}^{*}(x, y)$ converges and $|\psi(x, y)| \leqslant \varepsilon^{2}$.
Thus

$$
\Psi(I) \leqslant \varepsilon^{2} \text { meas } I \text { whence } \mathrm{V}\left(\Psi ; S_{0}\right) \leqslant \varepsilon^{2} \leqslant \frac{\varepsilon}{2} \cdot V_{0},
$$

and in view of $(2.9)$ we have $\mathrm{V}\left(\Phi ; S_{0}\right) \leqslant(1+\varepsilon) \cdot V_{0}$, which is the relation (b) of Theorem $B$. Also from the uniform convergence of the series (2.8) and from the first relation (2.10), it follows that $\Phi(I)$ is a continuous additive function of intervals.

It remains to prove that

(2.11) $\Phi^{*}(x, y)=\varphi(x, y)$ almost everywhere in $S_{0}$.

For this purpose, let $(x, y)$ be an arbitrary point in $A$. Since $\left\{E_{k}\right\}$ is a non-decreasing sequence, there exists a positive integer $k_{0}$ such that for $k \geqslant k_{0}$ the point $(x, y)$ belongs to any set $E_{k}$ while to no set $Q_{k}$. Therefore, in view of $\left(P_{4}\right)$, for every $k \geqslant k_{0}$ and every interval $I$ which contains $(x, y)$

$$
\left|\frac{F(I)}{\operatorname{meas} I}-\sum_{n=1}^{k} \frac{F_{n}(I)}{\operatorname{meas} I}\right| \leqslant\left|\sum_{n=k+1}^{\infty} \frac{F_{n}(I)}{\operatorname{meas} I}\right| \leqslant \frac{1}{2^{k}}
$$

passing to the linit as $\mathrm{d}(I) \rightarrow 0$ and $k \rightarrow \infty$, we receive thus

$$
F^{*}(x, y)=\sum_{n=1}^{\infty} F_{n}^{*}(x, y)=\varphi(x, y)-\psi(x, y)
$$

for any point $(x, y)$ in $A$, and so, on account of $\left(\mathrm{P}_{1}\right)$ and $\left(\mathrm{P}_{2}\right)$, almost everywhere in $S_{0}$. On the other hand, as $\psi(x, y)$ is bounded in $S_{0}$ (except perhaps on a set of measure zero), we have $\Psi^{*}(x, y)=$ $=\psi(x, y)$ almost everywhere $\left.{ }^{11}\right)$; which together with (2 12) establishes the relation (2.11) and completes the proof of Theorem $B$.

3. Remarks. To the theorem established in the preceding section a few remarks may be added. It is easily seen that the function $\Phi(x, y)$ subject to the conditions (a) and (b) of Theorem B may be defined so as to satisfy some more conditions of regularity; e. g. it may be supposed to be a sum of two functions $\Phi_{1}(x, y)$ and $\Phi_{2}(x, y)$ of which $\Phi_{1}(x, y)$ is an indefinite integral of a bounded function, and $\Phi_{2}(x, y)$ has continuous partial derivatives of the first order $\left.{ }^{12}\right) \partial \Phi_{2} / \partial x, \partial \Phi_{2} / \partial y$ everywhere in $S_{0}$ and those of the

12) It may be even supposed that $\Phi_{2}(x, y)$ has continuous partial derivatives $\partial^{k} \Phi_{2} / \partial x^{k}, \partial^{k} \Phi_{2} / \partial y^{k}$ of all orders. 
second order $\partial^{2} \Phi_{2} / \partial x \partial y=\partial^{2} \Phi_{2} / \partial y \partial x$ almost everywhere. Then the function $\Phi(x, y)$ almost everywhere possesses the derivatives $\partial \Phi / \partial x$ and $\partial \Phi / \partial y$, as well the derivatives $\partial^{2} \Phi / \partial x \partial y, \partial^{2} \Phi / \partial y \partial x$, with the restriction, however, that the latter are to be understond with respect to the set only on which $\partial \Phi / \partial x$ and $\partial \Phi / \partial y$ exist; so the condition (a) of Theorem B may be replaced by

(3.1) $\Phi^{*}(x, y)=\frac{\partial^{2} \Phi}{\partial x \partial y}=\frac{\partial^{2} \Phi}{\partial y \partial x}=\varphi(x, y)$ almost everywhere in $S_{0}$.

We shall also observe that, given an arbitrary enumerable set $N$ in $S_{0}$, the function $\Phi$ may be so constructed that the relation in (3.1) be satisfied, in particular, at every point of the set $\left.{ }^{18}\right) N$.

\section{References.}

1. Besicovitch, On differentiation of Lebesgue double integrals, Find. Math., this vol., pp. 209-216.

2. Busemann u. Fellex, Zur Differentiation des Lebesguesche Integrale, ibid, , vol. 22 (1934), pp. 226-256.

3. Jessen, Marcinkiewicz and Zygmund, Note on the differentiability of multiple integrals, ibid., this vol., pp. 217-234.

4. Looman, Sur la totalisation des dérivées des fonctions continues de plusieurs variables indépendantes, ibid., vol. 4 (1923), pp. 246-285.

5. Riesz F., Sur les points de densité au sens fort, ibid., vol. 22 (1934) pp. $221-225$.

6. $\mathrm{S}$ a $\mathrm{k}$, Remark on the differentiability of the Lebesgue indefinite integrat, ibid., vol. 22 (1934), pp. 20̄7-261.

7. Ward, On the differentiation of additive functions of reotangles, to appear in "Fundamenta".

8. $\mathrm{Z} \mathrm{ygmund}$, On the differentiability of multiple integrals, ibid, rol. 23 (1934), p. $143-149$

13) Cf. Eilenberg et $\mathrm{Saks}$, Sur la dérivation des fonctions dans des ensembles dénombrables, Fund. Math., this vol., p. 264--265.

\section{Über die stetigen Abbildungen der Strecke.}

Von

\section{Stefan Mazurkiewicz (Warszawa).}

Bezeichnungen. Sind $a, b$ reelle Zahlen, so bezeichnen wir mit $(a, b)$ das (atene mit $[a, b]$ das abgeschlossene Intervall mit den Endpunkten $a, b . R^{k}$ bezeichnet den $k$-ding ist $\sigma(A, B)=$ inf $\sigma(P, Q)$, wo $P \theta$ Diamer Pankteng and $Q \in B, \delta(A)$ der Diameter der Punktmenge $A \subset R^{k}$; ist $A \subset R^{k}, \lambda>0$, so bezeichnet $U(A, \lambda)$ die Menge aller Punkte $P \in R^{k}$, die der Ungleichung $\sigma(P, A)<\lambda$ genügen. $C^{k}$ bezeichnet die Menge der in $[0,1]$ definierten und stetigen Funktionen $f$, die der Bedingung $f(t) \in R^{k}$ genïgen. $C^{k}$ wird durch die Formel: $\varrho(f, g)=$ $=\operatorname{Max} \sigma\left(f(t), g\left(t_{i}\right) ; f \in C^{k}, g \in C^{k}\right.$ metrisiert. Wenn $f \in C^{k}, 0 \leqq \alpha \leqq \beta \leqq 1$, so ist $0 \leq t \leq 1=1(t)$ mit $t \in[\alpha, \beta]$. Wenn $P \in f[0,1], Q \in f[0,1]$, so bezeichnet $\sigma_{f}(P, Q)$ die relative Entfernung von $P$ und $Q$ in Bezug anf $f\left[0,1{ }^{1}\right)$.

H. Jarník hat folgenden Satz bewiesen ${ }^{2}$ ):

Es gibt in $C^{2}$ eine Residualmenge $W^{2}$, so dass jedes $f_{\epsilon} W^{2}$ folgende Eigenschaft besitzt: ist $0 \leqq \alpha<\beta \leqq 1$, so ist jeder Punkt der Kurve $f[\alpha, \beta]$ ein irregulärer Punkt dieser. Kurve.

H. Knaster hat mir die Vermutung mitgeteilt, dass folgender Satz richtig ist.

Satz. Es gibt in $C^{2}$ eine Residualmenge $W^{*}$, so dass für $f \in W^{*}$ die Krurve $f[0,1]$ mit der Universalkurve von Sierpiński homöomorph ist.

1) Fund. Math. I, p. 167-169.

2) J a rník, Monutsh. f. Muth. Phys, 41, p. 408-423, insb. p. 408, Satz 3, 2 und p. $417-423$ 